\title{
diffusion-Fundidameritals.org
}

The Open-Access Journal for the Basic Principles of Diffusion Theory, Experiment and Application

\section{Human Mobility and Spatial Disease Dynamics}

\author{
Dirk Brockmann ${ }^{1}$, Vincent David ${ }^{1,2}$ and Alejandro Morales Gallardo ${ }^{1,2}$ \\ ${ }^{1}$ Engineering Sciences and Applied Mathematics, Northwestern \\ University, 2145 Sheridan Rd., Evanston IL, 60208, United States \\ ${ }^{2}$ Faculty of Physics, Georg-August-Universität, \\ Friedrich-Hund-Platz 1, 37077 Göttingen, Germany
}

\begin{abstract}
The understanding of human mobility and the development of qualitative models as well as quantitative theories for it is of key importance to the research of human infectious disease dynamics on large geographical scales.In our globalized world, mobility and traffic have reached a complexity and volume of unprecedented degree. Long range human mobility is now responsible for the rapid geographical spread of emergent infectious diseases. Multiscale human mobility networks exhibit two prominent features: (1) Networks exhibit a strong heterogeneity, the distribution of weights, traffic fluxes and populations sizes of communities range over many orders of magnitude. (2) Although the interaction magnitude in terms of traffic intensities decreases with distance, the observed power-laws indicate that long range interactions play a significant role in spatial disease dynamics. We will review how the topological features of traffic networks can be incorporated in models for disease dynamics and show, that the way topology is translated into dynamics can have a profound impact on the overall disease dynamics. We will also introduce a class of spatially extended models in which the impact and interplay of both spatial heterogeneity as well as long range spatial interactions can be investigated in a systematic fashion. Our analysis of multiscale human mobility networks is based on a proxy network of dispersing US dollar bills, which we incorporated in a model to produce real-time epidemic forecasts that projected the spatial spread of the recent outbreak of Influenza A(H1N1).
\end{abstract}

Keywords: multiscale human mobility networks, emergent infectious diseases, spatially extended epidemic models, fractional diffusion, spatial heterogeneity 


\section{Introduction \& Motivation}

The understanding of human mobility and the development of qualitative models as well as quantitative theories for it is of key importance to the research of human infectious disease dynamics on large geographical scales. Grenfell et al. state [1]:

"Spatial transmission of directly transmitted infectious diseases is ultimately tied to movement by the hosts. The network of spatial spread (the disease's spatial coupling) may therefore be expected to be related to the transportation network within the host metapopulation."

In our globalized world, mobility and traffic have reached a complexity and volume of unprecedented degree. More than 60 million people travel billions of miles on more than 2 million international flights each week, see e.g. Fig. 2. Hundreds of millions of people commute on a complex web of highways and railroads most of which operate at their maximum capacity. Despite this increasing connectivity and our ability to visit virtually every place on this planet in a matter of days, the magnitude and intensity of modern human traffic has made human society more susceptible to threats intimately connected to human travel. Long range human mobility is now responsible for the rapid geographical spread of emergent infectious diseases. One of the prime examples of a modern epidemic is the severe acute respiratory syndrome (SARS) outbreak of 2003. Since then, an increasing amount of attention and modeling effort has been devoted to understanding to what extent modern traffic networks impact and determine the dynamics of emergent diseases. More recently, a novel strain of Influenza $\mathrm{A}(\mathrm{H} 1 \mathrm{~N} 1)$, also known as swine flu, first detected in Mexico and the United States, spread rapidly around the globe [2].

Consequently, intense research effort has been devoted during the recent decade to the development of quantitative models for the spread of human infectious diseases. In the past even the most sophisticated models had to make plausible assumptions on human interactions and their mobility, the key driving forces of an epidemic across distance. Presently, with increasingly availability of new data sources on human interactions and mobility, we observe a structural change in the development of these models. With quantitative assessments of human interactions and mobility computational epidemiology is now at the brink of developing models that are (1) predictive, (2) adaptive, and (3) flexible and that can be used as a foundation for the development of forecast infrastructures for disease dynamics.

A first step into this direction was recently tested in the context of the spread of swine flu with projections based on a computational model of the H1N1 spread in the US [3]. Figure 1 depicts the temporal evolution of the cases in a worst case scenario. The projected time course in these simulations agreed well with the time course that was observed later, and was a clear indication and motivation to elaborate, intensify and further develop these modeling approaches.

Needless to say, such a framework needs very detailed information describing human mobility patterns. In a number of recent studies the statistical properties of particular human transportation networks were investigated in detail with a focus 

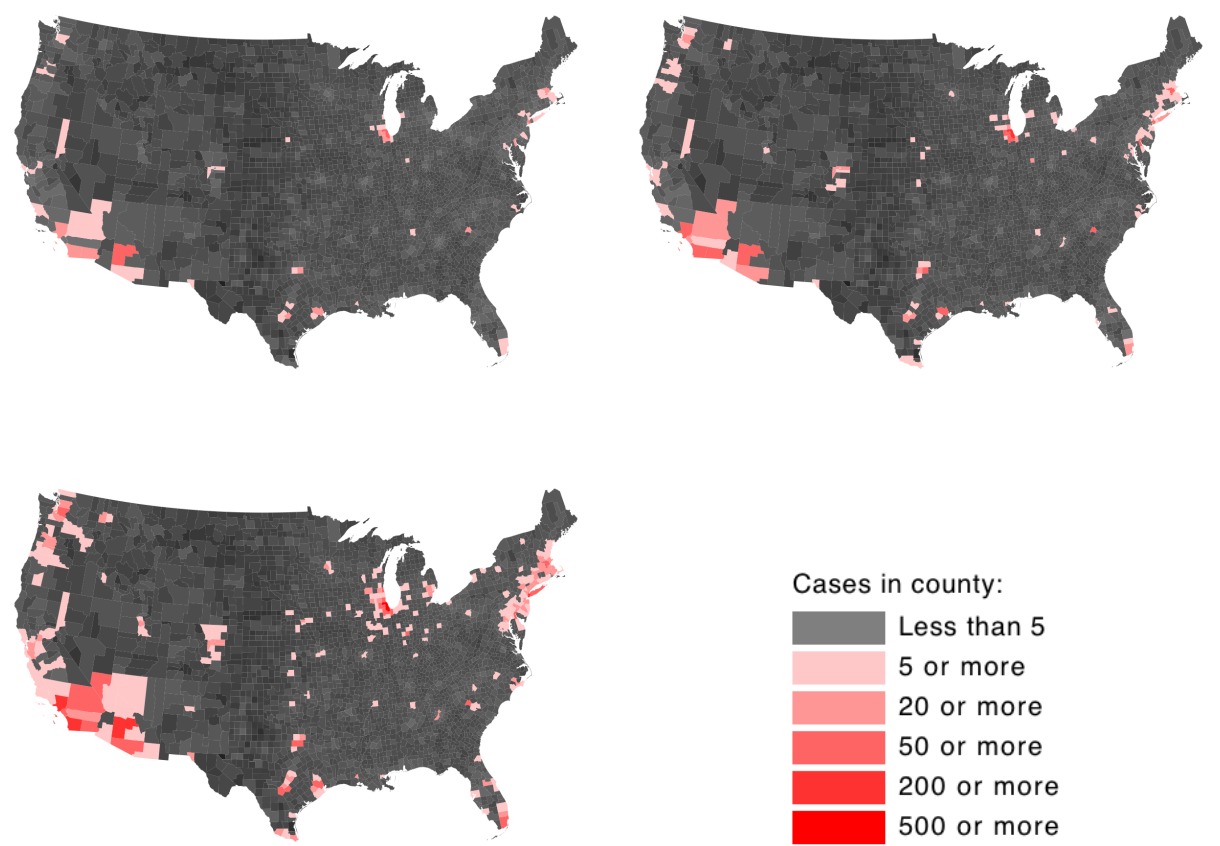

Figure 1: The first attempt of an "into the future" projection of the time course of an emergent infectious disease. These maps were computed with high performance computational techniques and multi-layer, large scale computer simulations to project the time course of a novel strain of Influenza $\mathrm{A}(\mathrm{H} 1 \mathrm{~N} 1)$ epidemic in the United States. The simulations yield projections and risk assessments of the epidemic outbreak in a worst case scenario, in which no containment measures are taken to mitigate the spread. The figures show the expected number of cases in each county on May 6, May 13, and May 20, 2009 using the confirmed cases as of May 6 to construct the initial condition. 


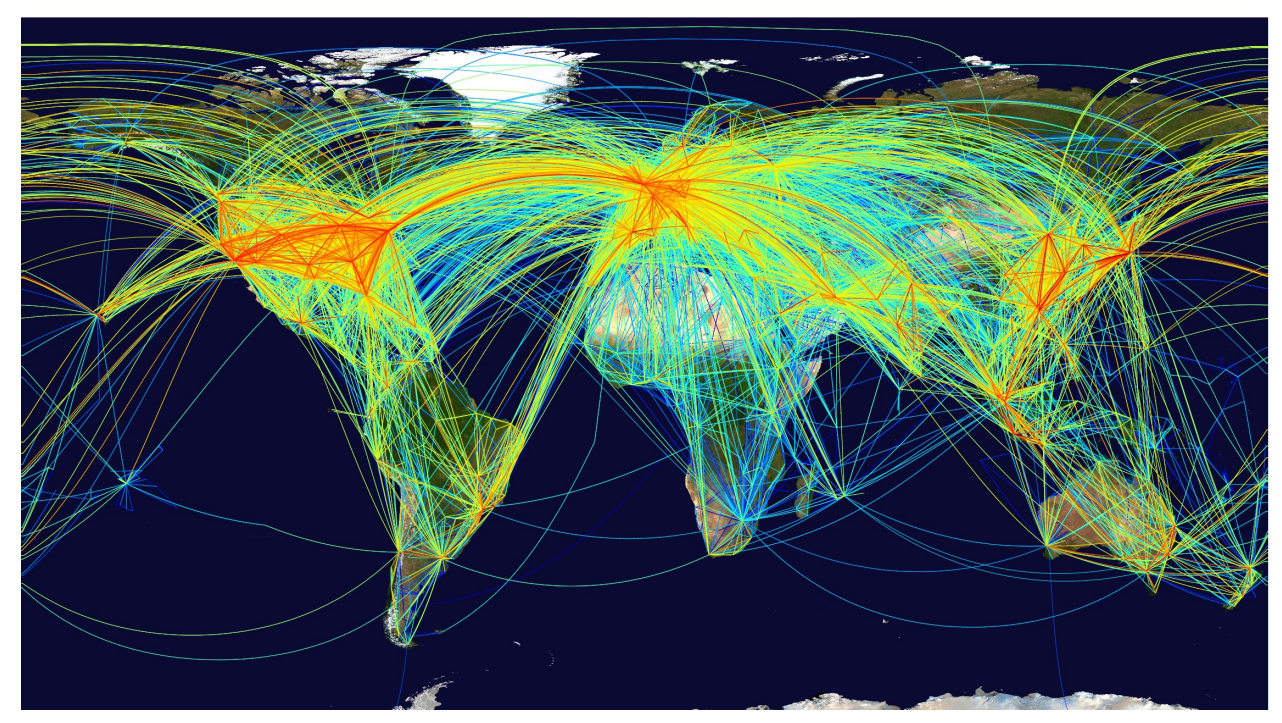

Figure 2: The worldwide air transportation network. More than 3 billion passengers travel on this network each year, on flights connecting approx. 4000 airports. The heterogeneity of the network is reflected by the flux of individuals between nodes, ranging from a few to more than 10,000 passengers per day between nodes.

on air transportation and long distance traffic $[4,5,6,7]$. However, human mobility occurs on many length scales, ranging from commuting traffic on short distances to long range travel by air, and involves diverse methods of transportation (public transportation, roads, highways, trains, and air transportation). A comprehensive study incorporating traffic on all spatial scales into a multi-scale dataset would be a difficult task, furthermore the statistical features of such study are still not fully understood. How do these properties depend on the length scale? Are they universal? In what way do they change as a function of length scale? What are the national and regional differences and similarities? In order to understand human mobility in the 21st century and the dynamics of associated phenomena, particularly the geographic spread of modern diseases, it is of fundamental importance to answer these questions.

With this in mind, we have recently created a model from the multi-scale transportation network and methods to be discussed in this chapter and used it to produce real-time epidemic forecasts that projected the spatial spread of the recent outbreak of Influenza $\mathrm{A}(\mathrm{H} 1 \mathrm{~N} 1)$ in the United States over a course of up to three weeks (see Fig. 1). Critical geographical regions or hotspots can be identified on time (to help mitigate the impact of the disease) and the framework allows to flexibly adapt to the unraveling of new events in new projections by updating initial conditions or recalibrating model parameters. Furthermore, changes in mobility 
patterns due to travel restrictions can be addressed.

Epidemic models have been devised in the past on a wide range of complexity levels. On one end of the spectrum are reaction diffusion models in which local nonlinear infection dynamics is coupled with diffusive dispersal. Spatial heterogeneity in the host population is generally neglected in these models[8]. Questions they address are for example: Under what circumstance does a propagating epidemic wave develop? How does the speed of the wave depend on the parameters of the model? What impact does spatial heterogeneity have on the disease dynamics, and what are the statistical regularities in spatial patterns?

On the other end of the spectrum are sophisticated models that are constructed with a high degree of detail $[9,10,11,12]$. Examples of these models are agent based simulation frameworks in which social, spatial and temporal heterogeneity are taken into account. Frequently these models contain entire global transportation networks and extrapolations where empirical data is lacking based on known statistics.

This chapter contains three parts. In the first two we will discuss quantitative assessments of human mobility and recent progress in the study of multi length scale transportation networks. We will show that despite their complexity these networks exhibit a set of scaling relations and statistical regularities. In the last part we will review how the topological features of traffic networks can be incorporated in models for disease dynamics and show that the way topology is translated into dynamics can have a profound impact on the overall disease dynamics.

\section{Quantitative Assessments of Human Mobility}

\subsection{Preliminary Considerations}

Formally we can address the issue of mobile individuals by the collection of individual trajectories of each of $\mathcal{N}$ individuals of a population, i.e. the collection $\left\{\mathbf{x}_{i}(t)\right\}_{i=1, \ldots, \mathcal{N}}$ where each individual is labeled $i$. Clearly, the measurement and the prediction of each individual's location $\mathbf{x}_{i}(t)$ as a function of time is beyond a researcher's grasp. Some very recent experiments, however, employing high precision measurements based on GPS (global positioning via satellite) or using cell phone location as a proxy for $\mathbf{x}_{i}(t)$ have made it possible to measure - at least to some extent - individual trajectories with unexpected accuracy[13]. The next best approach to human mobility is based on population averages. To this end it is useful to define the microscopic time dependent density of individuals

$$
u(\mathbf{x}, t)=\frac{1}{A} \sum_{i}^{\mathcal{N}} \delta\left(\mathbf{x}-\mathbf{x}_{i}(t)\right),
$$

where $A$ is the spatial area under consideration. The global density of individuals in $A$ is given by the integral of $u$, i.e.

$$
u_{0}=\frac{\mathcal{N}}{A}=\int \mathrm{d} \mathbf{x} u(\mathbf{x}, t) .
$$


The expectation value $\langle u(\mathbf{x}, t)\rangle$ of the microscopic density is related to the probability $p_{i}(\mathbf{x}, t)$ of individual $i$ being located at $\mathbf{x}$ by

$$
\begin{aligned}
\langle u(\mathbf{x}, t)\rangle & =\frac{1}{A} \sum_{i}^{\mathcal{N}}\left\langle\delta\left(\mathbf{x}-\mathbf{x}_{i}(t)\right)\right\rangle \\
& =\frac{1}{A} \sum_{i}^{\mathcal{N}} p_{i}(\mathbf{x}, t) .
\end{aligned}
$$

Because for each $i$ even the quantity $p_{i}(\mathbf{x}, t)$ is usually inaccessible to measurement, a widespread assumption made in models is that individuals are indistinguishable and that although $\mathbf{x}_{i}(t) \neq \mathbf{x}_{j}(t)$ one assumes $p_{i}(\mathbf{x}, t)=p_{j}(\mathbf{x}, t)$ and thus

$$
\langle u(\mathbf{x}, t)\rangle=\frac{1}{A} p(\mathbf{x}, t) .
$$

Albeit its simplicity, this equation is fundamental for the probabilistic interpretation of models that are based on the time-evolution of concentrations. It connects the probabilistic quantity $p(\mathbf{x}, t)$ to the measurable density of individuals. The second assumption in the conceptual setup of analysing human mobility is an ergodicity assumption, that is given by

$$
\frac{1}{\Delta A} \int \mathrm{d} A u(\mathbf{x}, t) \approx\langle u(\mathbf{x}, t)\rangle,
$$

in which $\Delta A \ll A$ is an area small in comparison to the spatial size of the entire system but large enough such that sufficiently many individuals reside in it at all times such that the spatial average (lhs. of Eq. (5)) is approximately equal to the expected density. The degree to which these assumptions are fulfilled determines the right choice of models. Two structurally different models reflect a range of possibilities.

On the one hand, if $p(\mathbf{x}, t)$ varies over a limited amount of scales in magnitude and the global density $\mathcal{N} / A$ is large enough, one can find a microscopic scale $\Delta A$ such that a sufficient amount of individuals is always contained in each microscopic unit area for (5) to be valid. On a large scale one can then consider

$$
n(\mathbf{x}, t)=\Delta A\langle u(\mathbf{x}, t)\rangle,
$$

a spatially continuous deterministic quantity, and introduce dynamical equations for it.

Humans, however, are typically clustered in urban areas, cities, towns and villages in which the density of individuals is high as opposed to areas in between where is it negligible. In this case a metapopulation approach is more suitable. In this approach communities are defined by $p(\mathbf{x}, t)$ exceeding some threshold in some spatially compact area $\Omega_{n}$ and one labels these regions by a discrete index $n$. The size of each community $n$ is given by

$$
N_{n}(t)=\Omega_{n}\langle u(\mathbf{x}, t)\rangle .
$$


In these models mobility of individuals is equivalent to exchange of them between the discrete set of communities. In metapopulation models $N_{n}(t)$ is typically considered a deterministic quantity for which (5) holds. The coupling of these communities is conveyed by mobility networks that quantify the exchange of individuals between them. Usually these traffic networks are quantified by a matrix $W_{n m} \geq 0$ whose elements reflect the traffic flux between communities.

\subsection{The Lack of Scale in Human Mobility}

By far the most studied human mobility system, particularly in the context of human infectious disease dynamics, is the worldwide air transportation system, see Fig. 2. The network is defined by a passenger flux matrix, each element $W_{n m}$ of which quantifies the number of passengers that travel between airport $m$ and $n$. In a series of studies air transportation networks were investigated with methods of complex network theory[7, 4, 14] and have been employed as the backbone in a set of models that attempt to account for the global spread of emergent human infectious diseases $[15,6,5]$.

However, one of the central drawbacks of focusing on air-transportation alone is that only long range traffic is covered by it. If for instance one sets out to develop a model for disease dynamics on small to intermediate length scales, e.g. in countries such as Germany or the UK, air transportation does play a role, but an insignificant one compared to traffic on the network of highways and railways. Confronted with the difficulty of compiling a comprehensive dataset of human mobility covering all length scales, recently the idea was developed to employ proxies of human travel that indirectly provide information on mobility patterns of individuals. In Ref.[16] this idea was employed for the first time by analyzing the geographical circulation of bank notes. In the study, data was analyzed that was collected at the online bill tracker www.wheresgeorge.com founded by Hank Eskin in 1998. The idea of the game is simple: Individual dollars bills are marked and enter circulation. When new users come into possession of a marked bill, they can register at the site and report the current location of the bill by entering the zip code. Successive reports of a bill yield a spatio-temporal trajectory with a very high resolution. Since 1998 wheresgeorge.com has become the largest bill tracking website worldwide with more than 3 million registered users and more than 140 million registered bills. Approx. 10\% of all bills have been reported at least once after entry into the database, yielding a total of more than 14 million single trajectories consisting of origin $\mathbf{X}_{1}$ (initial entry location) and destination $\mathbf{X}_{2}$ (hit location). Figure 3 illustrates a sample of trajectories of bills with initial entries in five US cities. Shown are journeys of bills that lasted a week or less. Clearly, the majority of bills remains in the vicinity of their initial entry, yet a small but significant number of bills traversed distances of the order of the size of the US, consistent with the intuitive notion that short trips occur more frequently than long ones. One of the key results of the 2006 study was the first quantitative estimate of the probability $p(r)$ of a bill traversing a distance $r$ in a short period of time, a direct estimate of the probability of humans performing 

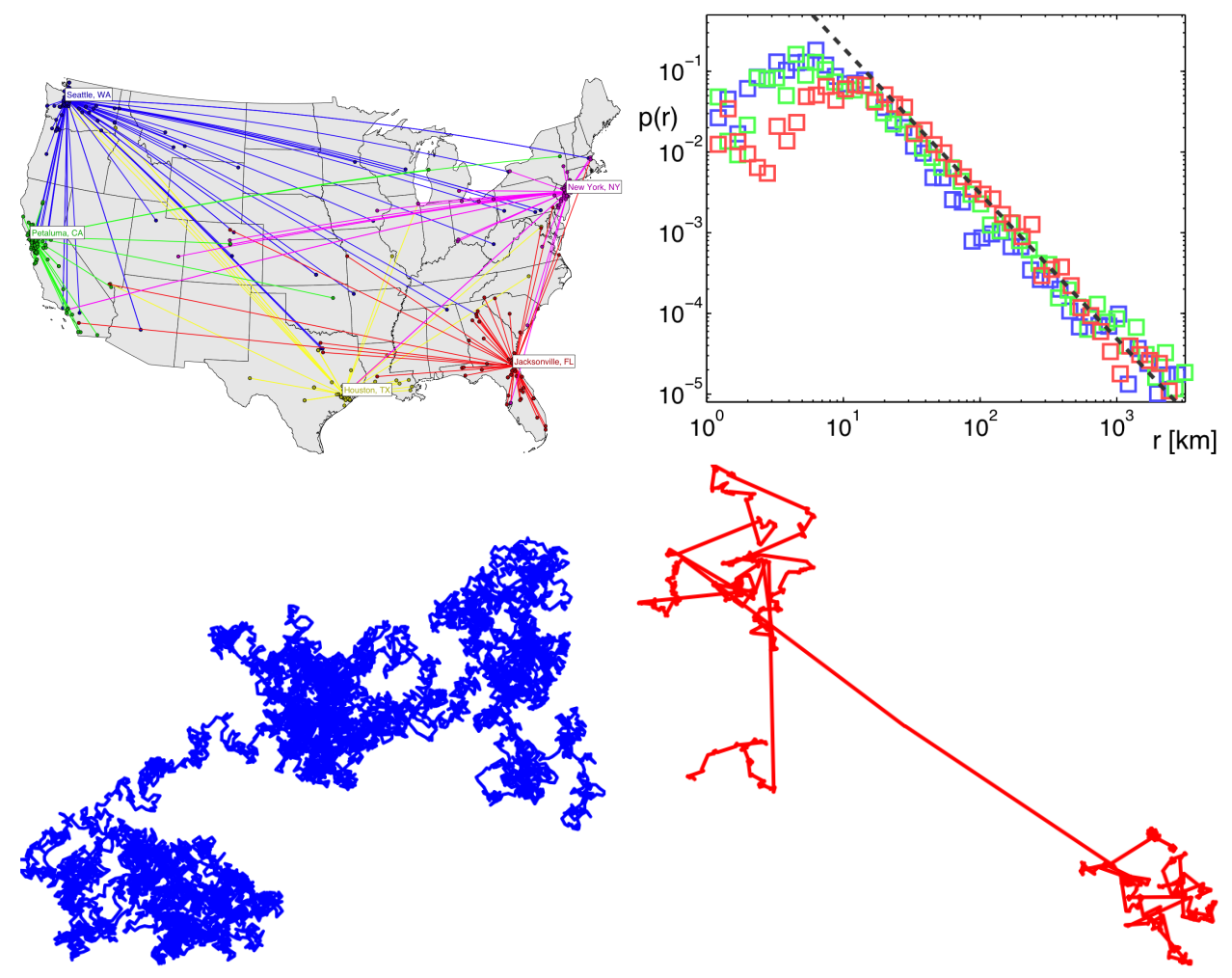

Figure 3: Short-time trajectories of dollar bills in the United States. Upper Left: Lines connect origin and destination locations of bills that traveled for less than a week. The majority of bills remain in the vicinity of their starting point, yet a small but significant fraction of bills travel long distances. Upper Right: The probability $p(r)$ of traveling a distance $r$ in a short period of time of $T$ less than a week. The dashed line indicates the inverse power law of Eq. (8) in the text. The colors encode the subsets of trajectories that started in large cities (blue), intermediate cities (green) and small towns (red). Despite systematic deviations for small distances, the asymptotic power law behavior is the same for all subsets indicating the universality of dispersal. Lower Left: Two dimensional trajectory of an ordinary random walk or Brownian motion. Lower Right: Trajectory of a superdiffusive Lévy flight. The Lévy flight geometry consists of small clusters interconnected by long leaps. The dispersal of bank notes is reminiscent of Lévy flight trajectories such as the one depicted. 
journeys of this distance in a short period of time. This quantity is shown in Fig. 3. This estimate was based on a dataset of 464,670 individual bills. On a range of distances between 10 and $3,500 \mathrm{~km}$, this probability follows an inverse power law, i.e.

$$
p(r) \sim \frac{1}{r^{1+\mu}},
$$

with an exponent $\mu \approx 0.6$. Despite the multitude of means of transportation involved, the underlying complexity of human travel behavior and the strong spatial heterogeneity of the United States, the probability follows this simple mathematical law indicating that human mobility is governed by underlying universal rules. Moreover the specific functional form has important consequences. If one assumes that individual bills perform a spatial random walk with an arbitrary probability distribution $p(r)$ for distances at every step one can ask: What is the typical distance $|\mathbf{X}(t)|$ from the initial starting point as a function of time? For ordinary random walks (Brownian motion) that are ubiquitous in the natural sciences, the

behavior of $|\mathbf{X}(t)|$ is determined by the standard deviation $\sigma=\sqrt{\left\langle r^{2}\right\rangle-\langle r\rangle^{2}}$ of the single steps and, irrespective of the particular shape of the distance distribution, scales according to the 'square root law', i.e. $|\mathbf{X}(t)| \sim \sqrt{t}$, a direct consequence of the central limit theorem[17]. However, for a power law of the type observed in the dispersal of bank notes the variance diverges for exponents $\mu<2$ and the situation is more complex. It implies that the dispersal of bank notes lacks a typical length scale, is fractal and the trajectories of bills are reminiscent of a particular class of random walks known as Lévy flights[18, 19]. Lévy flights, as opposed to ordinary random walks, are anomalously diffusive, they exhibit a scaling relation that depends on the exponent:

$$
|\mathbf{X}(t)| \sim t^{1 / \mu}
$$

Because Lévy flights are superdiffusive, they disperse faster than ordinary random walks, and their geometrical structure differs considerable from ordinary random walks, see Fig. 3. The discovery that the dispersal of bank notes and therefore human travel behavior lacks a scale and is related to Lévy flights was a major breakthrough in understanding human mobility on global scales.

\section{Statistical Properties and Scaling Laws in Multi Scale Mobility Networks}

Figure 4 illustrates a proxy network obtained from the flux of dollars in the United States, including all spatial scales. This network is defined by 3109 nodes (counties in the United States excluding Alaska and Hawaii) connected by weights $W_{n m}$ that represent the flux rate of bills from county $m$ to $n$ in units of bills per day. The entire network structure is thus encoded in the $3109 \times 3109$ flux matrix $\mathbf{W}$. As each location has a well-defined geographical position, this multi-scale US traffic 

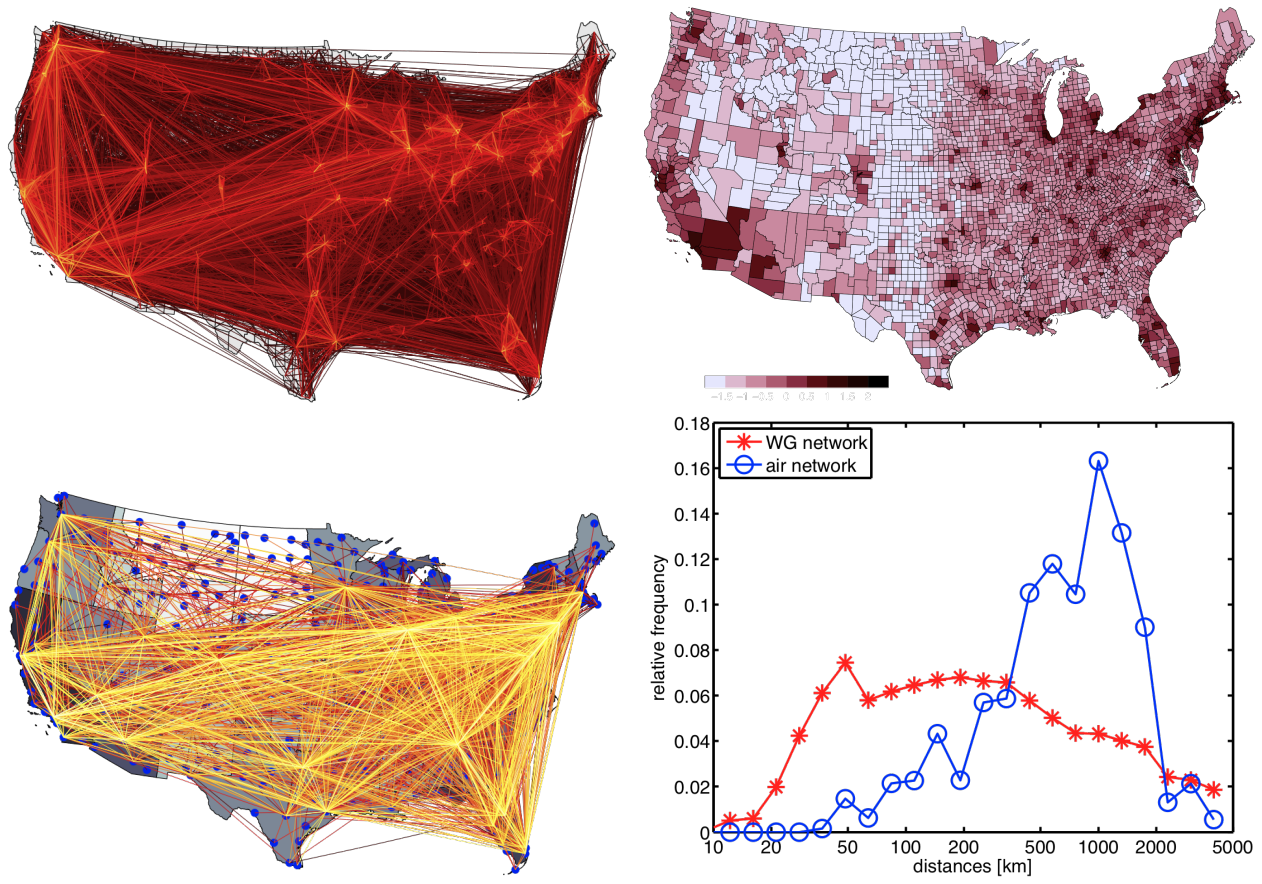

Figure 4: Top left: The flux of dollar bills in the United States. Each line represents the flux of bills between the counties it connects. The color encodes the magnitude of the flux, bright lines indicate heavy flux, dark lines weak flux. The figure illustrates the strong heterogeneity of money dispersal, short distance connections typically exhibit strong fluxes, long distance connections are weaker but significant. Upper Right: the population density of the United States spatially resolved and colored on a logscale. Lower left: The US air transportation network. The lines indicate connections between the 413 major airports in the US. The color encodes the magnitude of connections in passengers per day. Lower right: Relative frequency of distances in the multi-scale traffic network obtained from the Where's George dataset (red) compared to the air transportation network (blue). Air transportation mainly serves long distance whereas multi-scale traffic exhibits a broad distribution ranging from a few to a few thousand kilometers. 

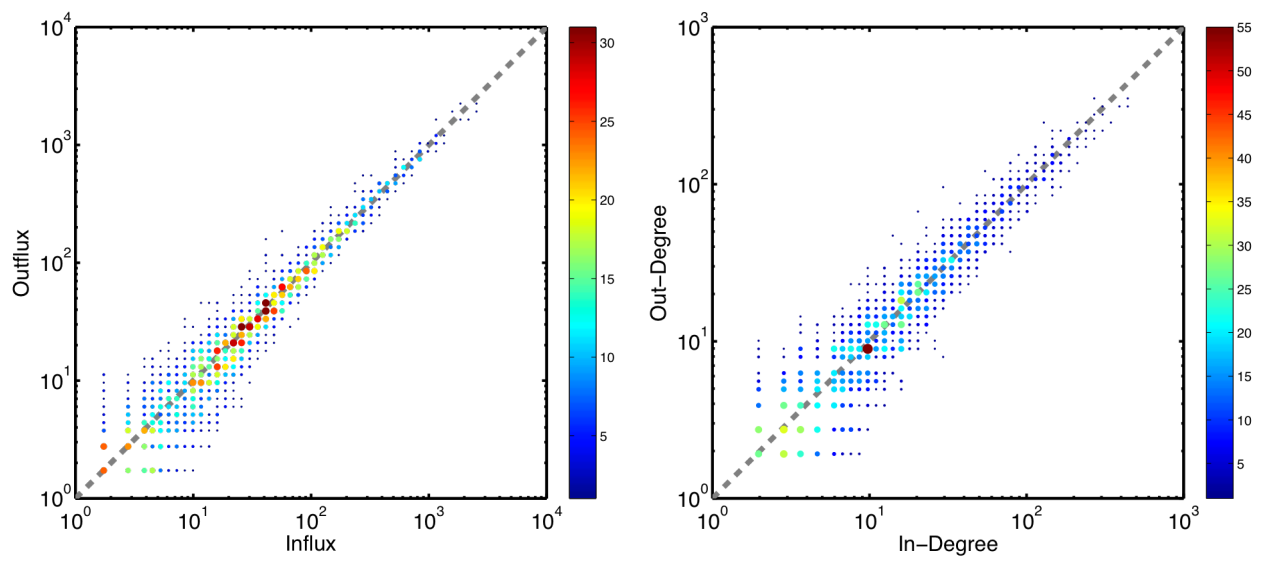

Figure 5: Symmetry of the money circulation network. The figures depicts the correlation $F_{n}^{\text {in }}$ and $F_{n}^{\text {out }}$ of flux of bill in and out and the in- and out degree $k_{n}^{\text {in }}$ and $k_{n}^{\text {out }}$ of a node $n$ for all 3109 nodes in the network. The dashed lines represent the linear relationships.

network can be visualized as a geographically embedded network as shown in the figure. Qualitatively, one can see that prominent East coast - West coast fluxes exist in the network. Yet the strongest connections are short to intermediate length scale connections, as opposed to the air transportation network that serves long distance only. Although every day 2.35 million passengers travel on the US air transportation network, this represents only a small subset of the multi-scale traffic network. The histogram in Fig. 4 illustrates these properties more quantitatively, comparing the relative frequency of distances in the multi-scale Where's George network compared to the air transportation network. Clearly, the majority of distances served by air transportation peaks around $1000 \mathrm{~km}$, whereas distances in the multi-scale network are broadly distributed across a wide range from a few to a few thousand kilometers.

In order to understand human mobility on all spatial scales it is therefore essential to include all means of transportation indirectly involved in the Where's George money circulation network. The bill circulation network quantified by the flux matrix can give important insight into the statistical features of human mobility across the United States. In order to quantify the statistical features of the network we will concentrate on the flux of bills in and out of a node given by

$$
F_{n}^{\text {in }}=\sum_{m} W_{n m} \quad F_{n}^{\text {out }}=\sum_{m} W_{m n}
$$

respectively. These flux measures are a direct proxy for the overall traffic capacity of a node in the network. Furthermore we will investigate the in- and out degree of 
a node defined according to

$$
k_{n}^{\text {in }}=\sum_{m} A_{n m} \quad k_{n}^{\text {out }}=\sum_{m} A_{m n},
$$

where the elements $A_{n m}$ are entries of the adjacency matrix $\mathbf{A}$. These elements are either one or zero depending on whether nodes are connected or not. The degree of a node quantifies the connectivity of a node, i.e. to how many other nodes a given node is connected. A first important but expected feature of the multi-scale mobility network is its degree of symmetry. Figure 5 depicts the correlation of the flux of bills in and out of each node and a correlogram of the in- and out-degrees. These quantities exhibit a linear relationship subject to fluctuations,

$$
F_{n}^{\text {in }} \approx F_{n}^{\text {out }} \quad k_{n}^{\text {in }} \approx k_{n}^{\text {out }},
$$

indicated by the dashed lines in the figure. Note also that the magnitude of the flux values ranges over nearly four orders of magnitude, a first indication of the strong heterogeneity of the network.
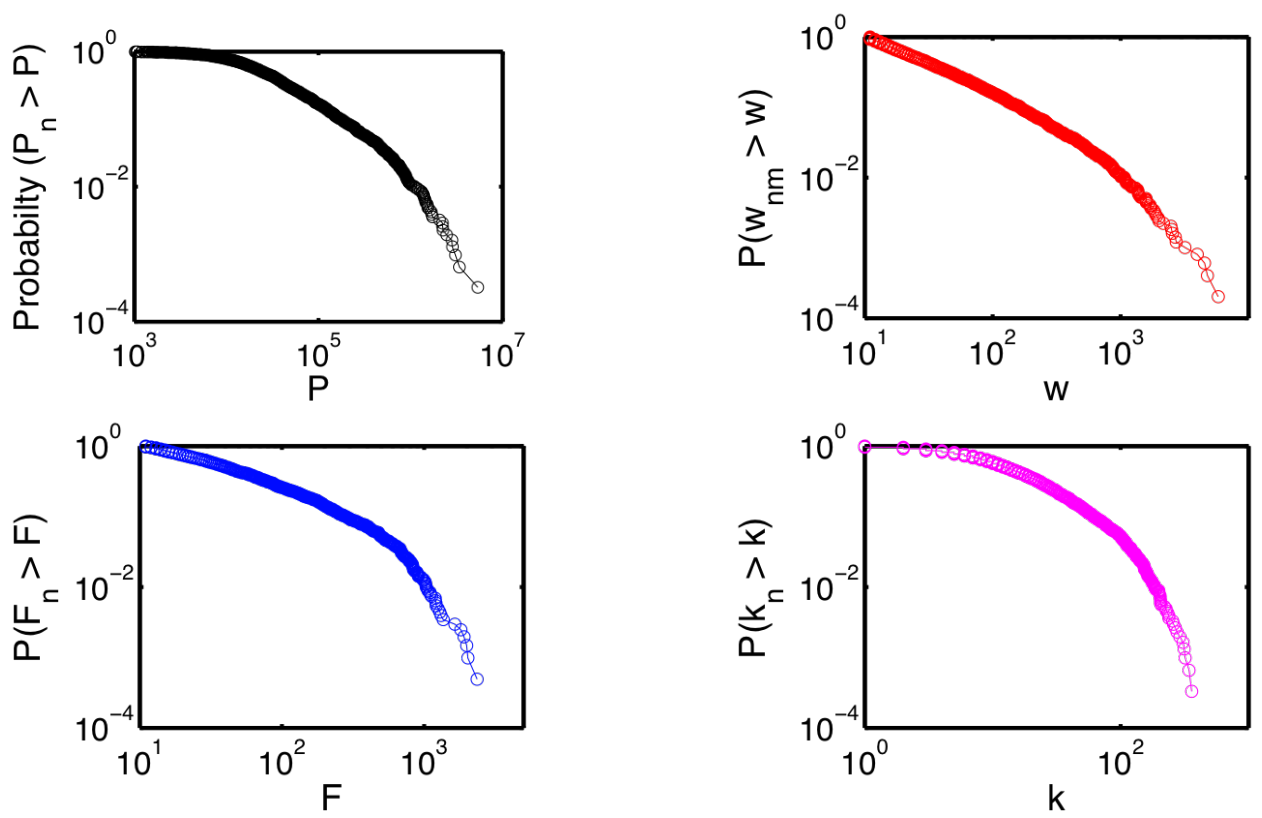

Figure 6: Heterogeneity of multi-scale human mobility networks. Cumulative probability distributions of the population size of the nodes (top left), the weight matrix elements $W_{n m}$ (top right), the flux of bills $F_{n}$ in and out of nodes (bottom left) and the degree $k_{n}$ of the nodes (bottom right). The broadness of these distributions is a consequence of the strong heterogeneity of the network. 
This high degree of heterogeneity is further illustrated by the cumulative distributions of the weights, the fluxes and the degrees of all the nodes in the network as depicted in Fig. 6. All quantities are broadly distributed across a wide range of scales. Very similar broad distributions have been observed in studies of the air transportation networks[7, 4, 14]. A very important issue in transportation theory is the development of a plausible evolutionary mechanism that can account for the emergence of these distributions, a task that has not been accomplished so far. There is no plausible 'theory' for human traffic networks as of today that predicts the precise functional form of the distributions shown in Fig. 6.

\section{Scaling Laws in the Topological Features of Multi-Scale Trans- portation Network}
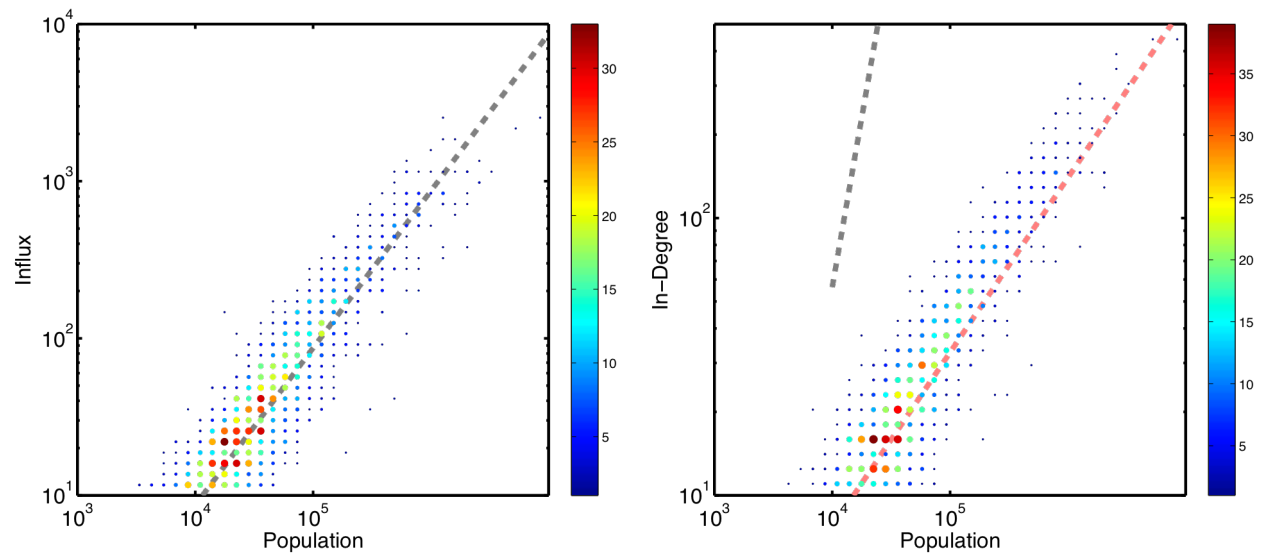

Figure 7: The functional dependence of influx $F^{\text {in }}$ (left) and in-degree $k^{\text {in }}$ (right) on the population size $P$ of a node. The flux of bills depends linearly on the population size (gray dashed line), whereas the degree exhibits a sublinear dependence (pink dashed line).

In order to reveal additional structure in multi-scale human mobility networks we investigated the functional relation of the quantities defined above, i.e.: What is the functional relation of fluxes and degrees with respect to the population size of a node? Figure 7 illustrates the statistical relationship between population size of a node and the flux of bills into a node. The dashed line in the figure represents a linear relationship with slope one, indicating that traffic through a node grows linearly with the population size:

$$
F(P) \sim P .
$$

Intuitively this is expected: the larger the population of a node the more traffic flows in and out of it. However, correlating the degree of a node against the population 
size indicates a sublinear relationship,

$$
k(P) \sim P^{\xi},
$$

with an exponent $\xi \approx 0.7$, contrasting the intuitive notion that the connectivity of a node grows linearly with population size as well. From the scaling relations (13) and (14) we can determine an important property of multi-scale mobility networks. The typical strength of a connection is given by the ratio of flux and degree and one obtains heuristically

$$
W \sim P^{1-\xi} .
$$

This implies that larger counties are not only connected to a larger number of other counties but also that the typical strength of every connection is stronger. Both relations are determined by the universal exponent $\xi=0.7$ and these relations hold over nearly four orders of magnitude, a surprising regularity exhibited by the multiscale mobility network. Again, no theory exists that can account for these scaling relations and the value of the exponent.

\section{Spatially Extended Epidemic Models}

In summary, two prominent features of multiscale human mobility networks emerged in the analysis above: (1) Networks exhibit a strong heterogeneity, the distribution of weights, traffic fluxes and population sizes of communities range over many orders of magnitude. (2) Although the interaction magnitude in terms of traffic intensities decreases with distance, the observed power-laws indicate that long range interactions play a significant role in spatial disease dynamics. In the models to be discussed below, we will introduce a class of spatially extended models in which the impact and interplay of both spatial heterogeneity as well as long range spatial interactions can be investigated in a systematic fashion. It will also become clear that another key issue in spatial disease dynamics is the translation of topological features of transportation networks, i.e. the flux matrix $\mathbf{W}$ into dynamical entities that generate the dispersal in space. At first glance, this may seem to be a straightforward process. However, as we will see, this is a nontrivial task, and the behavior of a spatially extended epidemic model depends sensitively on the precise choice of translating the topology of a transportation network into dynamics. To understand this, we first review some of the paradigmatic models for disease dynamics in a single population.

\subsection{Disease Dynamics in a Single Population}

One of the simplest models for an epidemic in a single population is the SIR model[20]. In this model a population of $N$ individuals is classified according to infectious state, i.e. a person can be susceptible (S) to the disease, infected (I) by the disease, and recovered $(\mathrm{R})$ from the disease. Recovered individuals are assumed 
to have acquired immunity to the disease and can no longer be infected. Each individual in a population may undergo the transition

$$
S \rightarrow I \rightarrow R
$$

during the time course of an infection. The dynamics of an epidemic is governed by only two reactions:

$$
\begin{array}{rlr}
S+I & \stackrel{\alpha}{\longrightarrow} & 2 I, \\
I & \stackrel{\beta}{\rightarrow} & R,
\end{array}
$$

a contact-initiated disease transmission and the recovery from disease, respectively. Models of this type are known as compartmental models, because a population is divided into different compartments defining the state of the system and reactions between individuals of various compartments define the dynamics. A key premise in the SIR model - and in fact most single population compartmental models - is the mixing assumption. It means that (1) all individuals of a given class are identical in their behavior and (2) independent of one another and that (3) reactions between a given pair of individuals occurs with the same likelihood as a reaction of any other pair.

The structure of compartmental models is very similar in nature to chemical reactions, in fact one usually employs the mass-action principle to derive ordinary differential equations for the dynamics of the number of susceptibles, infecteds and recovereds: At any point $t$ in time the probability that an infected individual recovers in $[t, t+\Delta t]$ is assumed to be constant and proportional to $\Delta t$. The change in infecteds and recovered is thus

$$
\Delta I=-\Delta R \approx-\beta \Delta t .
$$

The probability that an infected successfully transmits the disease to a susceptible in $\Delta t$ is given by

$$
P=\Delta t \times \sigma \times T \times \frac{S}{N},
$$

where $\sigma$ is the contact rate between individuals, $T$ the transmission probability and $S / N$ the probability that the contact is with a susceptible individual. This yields

$$
-\Delta S=\Delta I \approx \alpha \frac{S I}{N} \Delta t
$$

where $\alpha=\sigma T$ is the force of infection, i.e. the effective transmission rate. For the SIR-model this yields the following system of nonlinear ordinary differential equations (ODEs):

$$
\begin{aligned}
\partial_{t} S & =-\alpha \frac{S I}{N}, \\
\partial_{t} I & =\alpha \frac{S I}{N}-\beta I, \\
\partial_{t} R & =\beta I .
\end{aligned}
$$


We can define fractions $s=S / N, j=I / N$ and $r=R / N$ and noting that $S(t)+$ $I(t)+R(t)=N$ (i.e., the population size is conserved) we obtain the SIR model in its canonical form[21]:

$$
\begin{aligned}
\partial_{t} s & =-\alpha s j, \\
\partial_{t} j & =\alpha s j-\beta j, \\
r & =1-s-j .
\end{aligned}
$$

The key parameter in the SIR model is the basic reproduction number:

$$
R_{0}=\frac{\alpha}{\beta}=\frac{T_{\text {recovery }}}{T_{\text {contacts }}}
$$

the ratio of the force of infection and recovery rate. It is the average number of secondary infections caused by one infected individual during the time that individual is infected, on average. When $R_{0}>1$, a population with an initially small fraction of infecteds will be subject to an epidemic: a fast exponential increase and a subsequent decay of $j(t)$, see Fig. 8. When $R_{0}<1$ no epidemic occurs. The basic reproduction number is thus a threshold parameter.

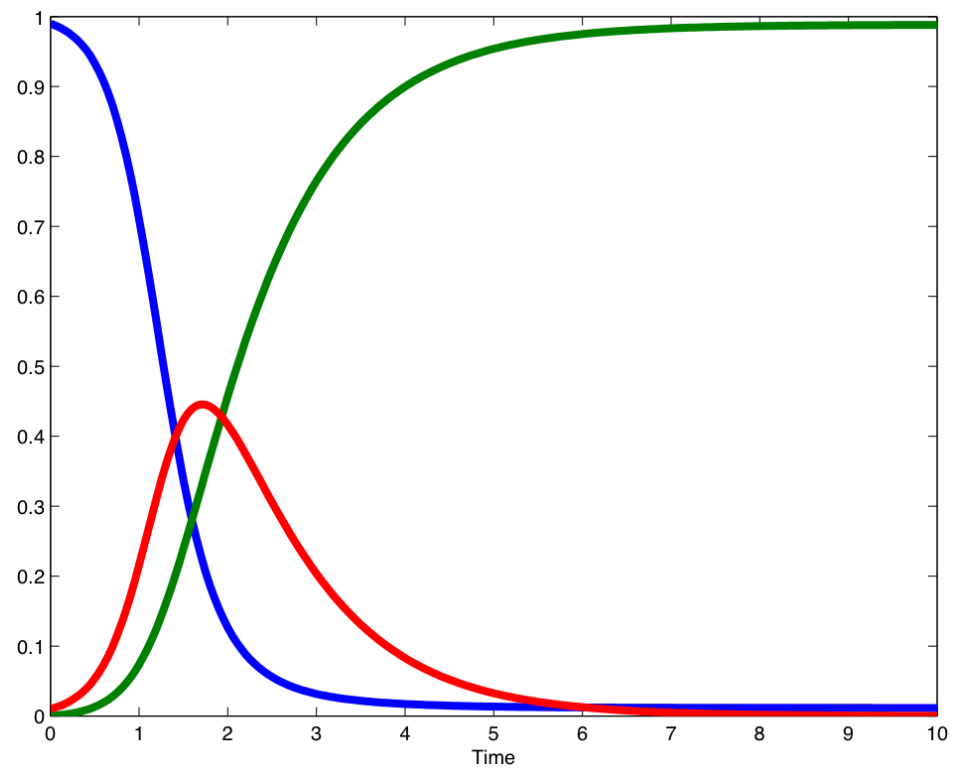

Figure 8: Time evolution of the SIR model as defined by Eqs. (23). Parameters are $\beta=1$ and $R_{0}=4.5$. Time course of the fraction of infecteds, susceptibles and recovereds are shown in red, blue and green respectively. The initial condition was $j(0)=0.01, s(0)=1-j(0)$ and $r(0)=0$. 


\section{The SIS model}

In the SIS model the second reaction scheme (18) is replaced by $I \rightarrow S$, infected individuals do not acquire immunity but rather recover from the disease to become susceptible again. This model lacks the $R$ class and is governed by only one ODE for the infecteds,

$$
\partial_{t} j=\alpha j(1-j)-\beta j,
$$

where the conservation of individuals $s=1-j$ is assumed. For $R_{0}=\alpha / \beta>1$ the SIS model evolves to a stable stationary state given by

$$
j^{s}=1-\frac{1}{R_{0}},
$$

in which a fraction $j^{s}$ of the population is infected, the disease is endemic. The SIS model is a useful system for investigating the impact of space on disease dynamics and we will discuss the spatially extended SIS model in the next section.

\subsection{Spatial Models}

In the heart of all spatial models is the motivation to forsake the assumption of homogeneous mixing of individuals and incorporate the fact that individuals belonging to different populations exhibit different interaction probabilities and that they are mobile in space. The conceptual tool underlying the development of spatial models is that of a metapopulation. A metapopulation is a set $m=1, \ldots, M$ of populations of size $N_{n}$. The total number of individuals of the metapopulation is

$$
N=\sum_{n=1}^{M} N_{n} .
$$

It is usually assumed that the dynamics in each population is governed by dynamics that adhere to homogeneous mixing but interaction of individuals between populations are governed by additional laws. The most important of such interactions for disease dynamics is the random exchange of individuals between populations. The most straightforward generalization of the SIS model including metapopulations is given by:

$$
\begin{array}{rll}
S_{n}+I_{n} & \stackrel{\alpha}{\longrightarrow} & 2 I_{n} \\
I_{n} & \stackrel{\beta}{\longrightarrow} & S_{n} \\
S_{n} & \stackrel{w_{m n}}{\longrightarrow} & S_{m} \\
I_{n} & \stackrel{w_{m n}}{\longrightarrow} & I_{m}
\end{array}
$$

In addition to the first two reactions, i.e. ordinary SIS dynamics in each population $n$, susceptibles and infecteds can randomly move between population $m$ and $n$, the rate of which is governed by the probability rate $w_{m n}$. The assumption in this 
model is that individuals of all types randomly travel between populations in the same fashion. The set of ODEs governing disease dynamics is then given by an set of $2 M$ coupled ODEs:

$$
\begin{aligned}
\partial_{t} S_{n} & =-\alpha \frac{S_{n} I_{n}}{N_{n}}+\beta I_{n}+\sum_{m \neq n}\left[w_{n m} S_{m}-w_{m n} S_{n}\right], \\
\partial_{t} I_{n} & =\alpha \frac{S_{n} I_{n}}{N_{n}}-\beta I_{n}+\sum_{m \neq n}\left[w_{n m} I_{m}-w_{m n} I_{n}\right] .
\end{aligned}
$$

The total rate of leaving a node $n$ is given by $\sum_{m \neq n} w_{m n}$ and the expected time an individual remains in a population $n$ is

$$
\left\langle T_{n}\right\rangle=\frac{1}{\sum_{m \neq n} w_{m n}} .
$$

Note that in the metapopulation system the number $N_{n}(t)=S_{n}(t)+I_{n}(t)$ of individuals in each subpopulation is generally time-dependent, in fact adding the ODEs pairwise we obtain

$$
\partial_{t} N_{n}=\sum_{m \neq n}\left[w_{n m} N_{m}-w_{m n} N_{n}\right]
$$

In most models it is usually assumed that the system is equilibrated with respect to dispersal, i.e. $N_{n}$ does not change over time and is therefore equal to the fixed point of Eq. (29), i.e.

$$
N_{n}(t)=N_{n}^{s}=C_{n}=\text { const. }
$$

In the following we will refer to the stationary population size of node $n$ as the capacity $C_{n}$. In equilibrium the flux of individuals from $n$ to $m$ balances that of $m$ to $n$ (detailed balance condition):

$$
w_{n m} C_{m}=w_{m n} C_{n} .
$$

In this case the spatial SIS model (27) reduces to a set of $M$ coupled ODEs for the fraction of infecteds in each population:

$$
\partial_{t} j_{n}=\alpha j_{n}\left(1-j_{n}\right)-\beta j_{n}+\sum_{m \neq n}\left[w_{n m} j_{m}-w_{m n} j_{n}\right]
$$

with $j_{n}=I_{n} / C_{n}$. The system defined by (32) is an example of an infectious disease dynamical system extended to the metapopulation level. A large class of contemporary models for spatial disease dynamics are related in structure to it $[5,22$, $15,9]$. One of the key difficulties in theoretical epidemiology are (1) the identification of effective communities of populations that make up a metapopulation and (2) the quantitative assessment of travelling rates $w_{n m}$ between these populations. Note that the introduction of populations $n$ making up the metapopulation did not specify 
spatial locations. In the dynamical system (32), the relation between communities is solely defined by the dynamical coupling $\omega_{n m}$. In most models, however, all communities are typically embedded in space such that each population $n$ has a well defined geographical location $\mathbf{x}_{n}$. One can then use the geographical information to make and test assumptions on how the exchange rates $w_{n m}$ depend on geography. One of the most popular assumptions in this context is that the flux of individuals between two communities depends on their size and their distance. The total flux of individuals in equilibrium from community $m$ to $n$ and vice versa is given by the left and the right hand side of the detailed balance condition (31), respectively. In the majority of models it is assumed that the flux $F_{n m}$ increases with the capacities (i.e. the stationary size of the populations) $C_{m}$ and $C_{n}$ and decreases monotonically with the geographic distance between them, i.e.

$$
F_{n m}=\omega_{0}\left(C_{m} C_{n}\right)^{\xi} G\left(\left|\mathbf{x}_{n}-\mathbf{x}_{m}\right|\right)=F_{m n},
$$

with $0 \leq \xi \leq 1$. The function $G$ takes care of the dependence on distance. Depending on the type of metapopulation and dynamical context, this kernel can be exponential, Gaussian or show an algebraic decay with $x$. Using the relation $F_{n m}=w_{n m} C_{m}$ between absolute flux and probability rates in equilibrium, Eq. (33) implies for the hopping rate

$$
w_{n m}=w_{0} C_{n}^{\xi} \times G\left(\left|\mathbf{x}_{n}-\mathbf{x}_{m}\right|\right) \times C_{m}^{\xi-1} .
$$

Inserted into the rate equation (29) one can check that $C_{n}$ is the equilibrium community size. In epidemiological contexts, spatial communities often reflect cities, towns and villages. The specific choice of $G(x)$ put forth by Eq. (8) is the power-law decay

$$
G(x) \sim x^{-1-\mu}
$$

Inserted into Eqs. (33) and (34) gives

$$
\omega_{n m}=\omega_{0} \frac{C_{n}^{\xi} \times C_{m}^{\xi-1}}{\left|\mathbf{x}_{n}-\mathbf{x}_{m}\right|^{D+\mu}},
$$

where $D=2$ is the spatial dimension. The parameter $\xi$ quantifies the impact of origin and destination in the travelling event $m \rightarrow n$ :

- When $\xi=1$ we have

$$
w_{n m} \propto C_{n} \text { and } F_{n m} \propto C_{n} C_{m} .
$$

This implies that the rate is independent of properties of the origin and the flux is proportional to the size of both communities.

- When $\xi=0$ we have

$$
w_{n m} \propto \frac{1}{C_{m}} \text { and } F_{n m} \propto 1
$$


i.e. the rate of traveling to destination $n$ is independent of properties of the destination and the flux is independent of community sizes of both places.

- An interesting system is the symmetric case when $\xi=1 / 2$. This implies that

$$
w_{n m} \propto \sqrt{C_{n} / C_{m}} \text { and } F_{n m} \propto \sqrt{C_{n} C_{m}} .
$$

In this situation, the rate $w_{n m}$ is independent of scaling the entire metapopulation size uniformly by some factor and the flux is the geometric mean of the community sizes of origin and destination. That implies for example: When we scale the entire population size by $C_{n} \rightarrow 2 C_{n}$ this scales the flux by a factor of two as well.

\subsection{Continuity Limit and Fractional Transport}

With the definition of the rate according to (36) the dispersal of individuals is given by

$$
\partial_{t} N_{n}=w_{0} \sum_{m \neq m}\left[\frac{C_{n}^{\xi} C_{m}^{\xi-1}}{\left|\mathbf{x}_{n}-\mathbf{x}_{m}\right|^{2+\mu}} N_{m}-\frac{C_{m}^{\xi} C_{n}^{\xi-1}}{\left|\mathbf{x}_{m}-\mathbf{x}_{n}\right|^{2+\mu}} N_{n}\right]
$$

with $\mu>0$. Useful insight into the properties of this master equation can be gained by performing a continuity limit. Letting $\mathbf{x}_{n}$ be points on a grid of microscopic areas $\Delta A$ and $N_{n}(t)=n\left(\mathbf{x}_{n}, t\right) \Delta A, C_{n}=c\left(\mathbf{x}_{n}\right) \Delta A$ the above equation becomes

$$
\partial_{t} n(\mathbf{x}, t)=w_{0} \lim _{\Delta A \rightarrow 0} \int_{\mathbf{y} \notin \Delta A} \mathrm{~d} \mathbf{y} \frac{c^{\xi}(\mathbf{x}) c^{\xi-1}(\mathbf{y}) n(\mathbf{y}, t)-c^{\xi}(\mathbf{y}) c^{\xi-1}(\mathbf{x}) n(\mathbf{x}, t)}{|\mathbf{x}-\mathbf{y}|^{2+\mu}} .
$$

The integral is over all points outside of an area centered at $\mathbf{x}$. One has to be careful carrying out this limit, because of the divergent denominator. In fact, originally the rate $m \rightarrow n$ was only defined for interacting communities $n \neq m$ and it is meaningless for $n=m$. One can, however, carry out the limit $\Delta A \rightarrow 0$ and interpret the integral as a Cauchy integral. The limit of the rhs. of (40) then depends sensitively on the value of the exponent $\mu$. For $\mu>2$ one obtains[23, 24]

$$
\partial_{t} n=D_{0}\left[c^{\xi} \Delta c^{\xi-1} n-c^{\xi-1} n \Delta n^{\xi}\right],
$$

with $n=n(\mathbf{x}, t)$ and $c=c(\mathbf{x})$ and $\Delta$ is the second spatial derivative. This implies that when the exponent $\mu$ exceeds the critical value $\mu_{c}=2$ the process becomes a diffusion process in the limit above. However, this diffusion process evolves in a heterogeneous environment determined by the function $c(\mathbf{x})$.

If $\mu<2$, as for example observed in the dispersal of bank notes (in that case $\mu \approx 0.6$ ), the limit yields

$$
\partial_{t} n=D_{0}\left[c^{\xi} \Delta^{\mu / 2} c^{\xi-1} n-c^{\xi-1} n \Delta^{\mu / 2} n^{\xi}\right]
$$


where the operator $\Delta^{\mu / 2}$ is known as the fractional Laplacian, a non-local singular operator defined by

$$
\left(\Delta^{\mu / 2} f\right)(\mathbf{x})=C_{\mu} \int \mathrm{d} \mathbf{y} \frac{f(\mathbf{y})-f(\mathbf{x})}{|\mathbf{x}-\mathbf{y}|^{D+\mu}}
$$

where $C_{\mu}$ is a constant and $D$ the spatial dimension[25, 26]. The reason why $\Delta^{\mu / 2}$ is referred to as a fractional derivative is that in Fourier space it exhibits a particularly simple form, a multiplication by $-|\mathbf{k}|^{\mu}$. Equations of the type (42) are known as fractional diffusion equations and have been employed in a number of physical, biological and chemical systems $[27,28,29,30]$, ranging from anomalous diffusion of protein motion on folded polymers to human eye-movements[31, 25, 26]. The derivation above relates dispersal of individuals in metapopulations to fractional diffusion equations for the first time, an approach that may well prove to be valuable in the future.

\subsection{Limiting Cases}

Before reinserting the dispersal component into the original spatial SIS model, it is worthwhile considering known marginal cases of the general fractional diffusion equation (42). For example when $\mu=2$ and $c(\mathbf{x})=1$ the dynamics equation reduces to

$$
\partial_{t} n=D_{0} \Delta n,
$$

i.e. ordinary diffusion in a homogeneous environment. When $\mu=2$ but $c(\mathbf{x})$ is a variable function of position, i.e. Eq. (42) is the same as Eq. (41), the dispersal is governed by a Fokker-Planck equation

$$
\partial_{t} n=-\nabla F n+\frac{1}{2} \Delta D n,
$$

which is equivalent to (41), and force and diffusion coefficients $F=F(\mathbf{x})$ and $D=D(\mathbf{x})$, respectively, are related to the heterogeneity function $c(\mathbf{x})$. This relation depends, of course, on the value of the parameter $\xi$. For example, when the system is origin-driven, i.e. when $\xi=0$, Eq. (41) reduces to

$$
\partial_{t} n=D_{0} \Delta n / c,
$$

a Fokker-Planck equation with a space dependent diffusion coefficient

$$
D(\mathbf{x})=\frac{D_{0}}{c(\mathbf{x})}
$$

that is inversely proportional to the stationary population density $c(\mathbf{x})$. This means that in this system diffusion is high in regions where the population is small and vice versa. In the destination driven system $\xi=1$, we obtain a Fokker-Planck equation with

$$
D(\mathbf{x})=2 D_{0} c(\mathbf{x}) \quad \text { and } \quad F(\mathbf{x})=2 D_{0} \nabla c(\mathbf{x}) .
$$


Here, diffusion increases with population density, but more importantly a nonzero drift towards regions with higher population density is introduced. When $\mu=1 / 2$, i.e. the impact of origin and destination are the same, the diffusion coefficient is constant and the force term is given by

$$
F(\mathbf{x})=D_{0} \nabla \log c(\mathbf{x}) .
$$

One can see that only in this situation the dynamics does not change when the population density $c(\mathbf{x})$ is scaled uniformly by some factor. In this case $-\log c(\mathbf{x})$ can be considered a potential $V(\mathbf{x})$ of the system, with minima in densely populated areas and maxima in weakly populated ones.

The most interesting case and certainly the one closest to reality is the general case, when the dynamical system is fractional diffusive and spatially heterogeneous. The combination of the rhs. of (42) with the spatial SIS model of Eq. (32) gives the spatially extended fractional SIS model,

$$
\partial_{t} j=\alpha j(1-j)-\beta j+D_{0} c^{\xi-1}\left[\Delta^{\mu / 2} c^{\xi} j-j \Delta^{\mu / 2} c^{\xi}\right] .
$$

The spatial SIR model or related systems that differ in the local dynamics can be derived analogously, for instance the spatial SIR model is given by

$$
\begin{aligned}
& \partial_{t} s=-\alpha j s+D_{0} c^{\xi-1}\left[\Delta^{\mu / 2} c^{\xi} s-s \Delta^{\mu / 2} c^{\xi}\right], \\
& \partial_{t} j=\alpha j s-\beta j+D_{0} c^{\xi-1}\left[\Delta^{\mu / 2} c^{\xi} j-j \Delta^{\mu / 2} c^{\xi}\right] .
\end{aligned}
$$

The key question is: What are the general properties of solutions to these reaction fractional diffusion equations? How do their solutions depend on the parameters $0 \leq \xi \leq 1$ and $0<\mu \leq 2$ ? And what are approximate choices for these parameters for real epidemics?

To address the first question, solutions of three variants of the spatial SIR model are depicted in Fig. 9. One system is spatially homogeneous and dispersal is ordinary diffusion. The solution exhibits traveling wavefronts that propagate at constant speeds, a fact known for similar systems such as the Fisher equation. In fact, a spatially homogeneous SIR variant was employed to estimate the speed of propagation of the black death in Europe in the 14th century. The second simulation is a system with some degree of spatial heterogeneity, i.e. $c(\mathbf{x})$ is variable but $\mu=2$. As in the spatial homogeneous system, solutions to the spatial SIR model still exhibit welldefined traveling wavefronts that exhibit some irregularity imposed by the spatial heterogeneity. However, the key feature of a wavefront propagating with a constant speed remains unchanged.

If however, one introduces non-local dispersal by choosing a value $\mu<2$, the overall statistical features of the spreading pattern changes drastically. Instead of a well shaped wavefront the pattern exhibits localized islands in the time course of the epidemic. This behavior is a direct consequence of the interplay of the spatial 

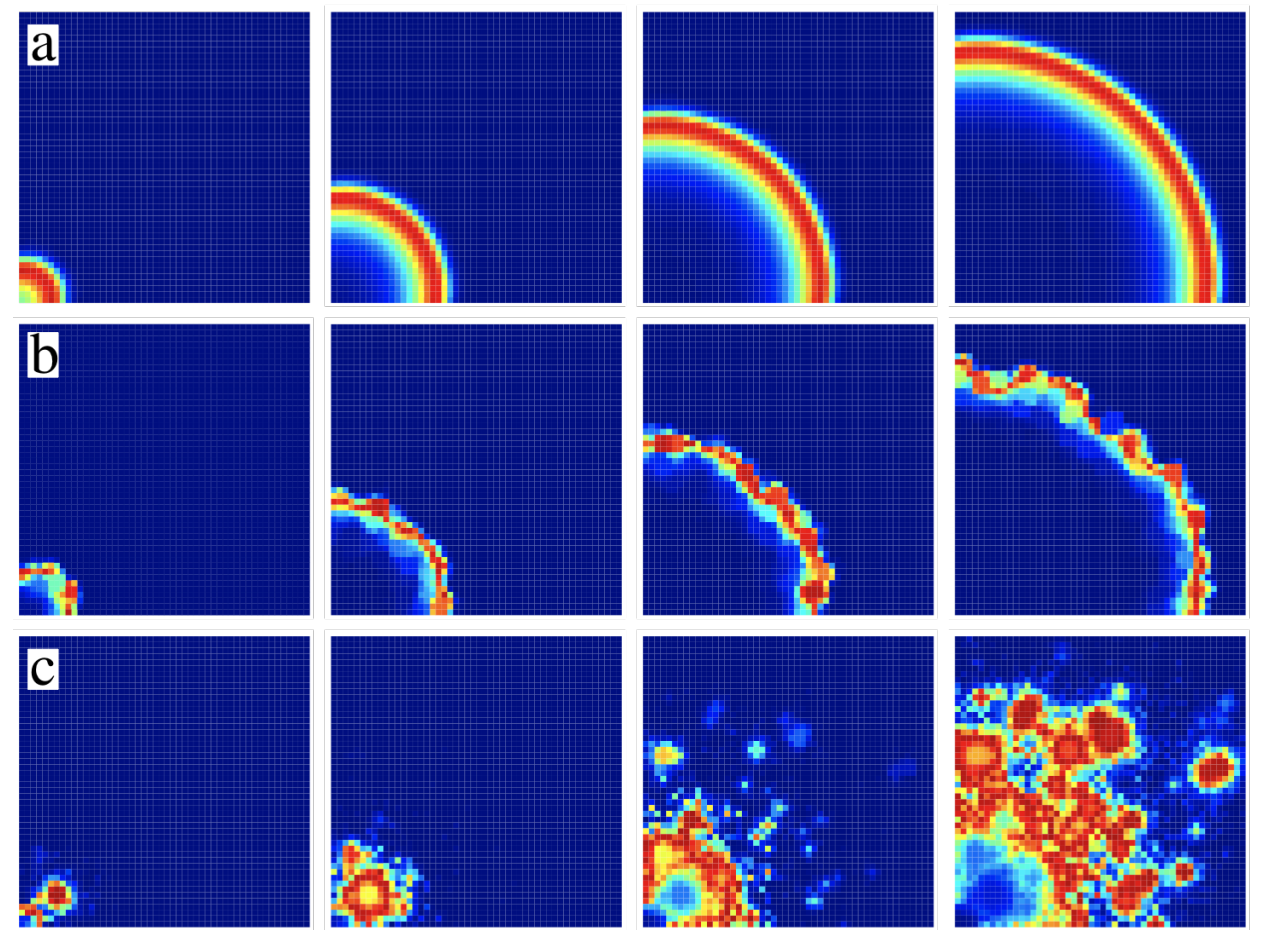

Figure 9: Snapshots of a two-dimensional spatially extended SIR model. Top: A spatially homogeneous system with $c(\mathbf{x})=$ const and ordinary diffusion in space. This system exhibits a propagating front at constant speed. Center: The same as above but with spatial heterogeneity. The heterogeneity induces randomness in the shape of the wavefront but produces no qualitatively different patterns. Bottom: The fractional SIR model with heterogeneity. The combination of scale-free diffusion and heterogeneity introduces a novel type of spatiotemporal pattern with fractal properties. 

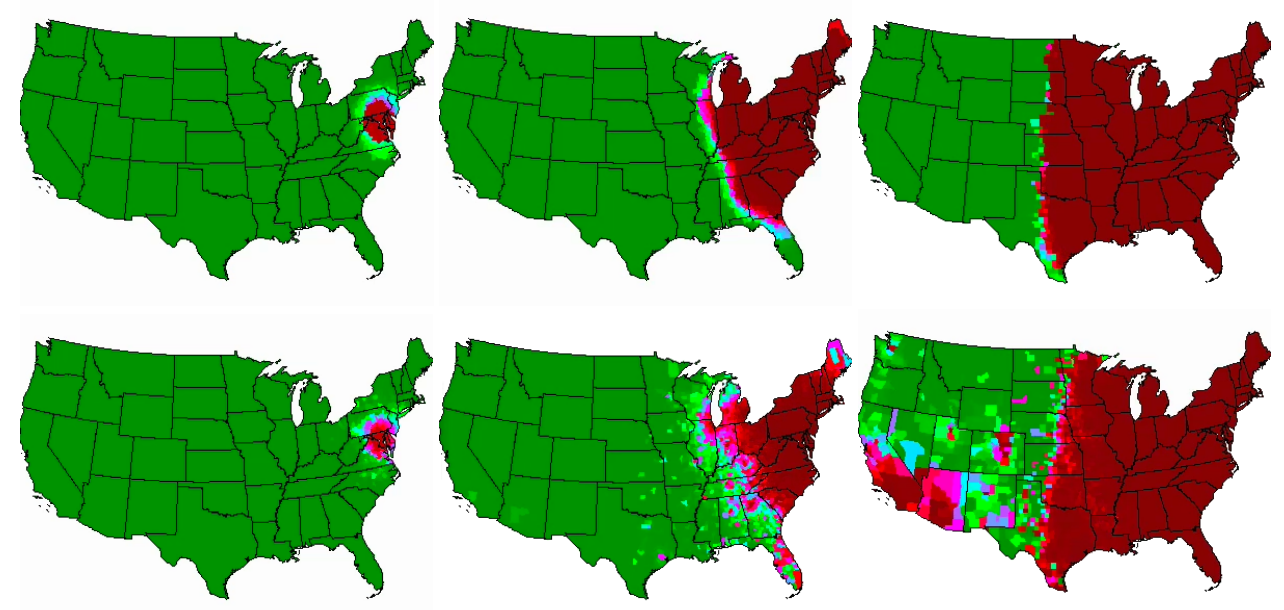

Figure 10: Simulation of the fractional spatially extended SIS model (bottom) in the United States compared to a system with ordinary diffusion (top). Each column represents a snapshot of the time evolution of both systems. Initial outbreak in the simulations was Washington, DC and parameters of the dispersal were $\xi=$ 1 (destination driven) and $\mu=0.6$ (superdiffusion, bottom) or $\mu=2$ (ordinary diffusion, top). The patterns exhibit the qualitative behavior of the idealized system shown in Fig. 9. 
heterogeneity and the non-local superdiffusive nature of dispersal incorporated in the fractional SIR model (51).

The last questions can be answered by a comparison to the empirical results presented above. The fact that the flux of dollar bills into nodes is proportional to the population size suggests that human dispersal is destination driven, see for example Eq. (37) and that $\xi=1$. The power-law in the short time dispersal probability for the distance, i.e. $p(r)$ in Eq. (8) implies that $\mu \approx 0.6$. With these parameters, and the equilibrium distribution of individuals in a large geographic area, we can investigate the spreading pattern in a real geographic context. Results are shown in Fig. 10 for a fractional SIS model with parameters $\mu=\mu_{h} \approx 0.6$ in the United States for an initial outbreak in Washington, DC. For $c(\mathbf{x})$ we chose the population density of the counties in the United States. In comparison to a system with only local dispersal, the fractional SIS systems shows a pattern similar in structure to the idealized system of a square grid (i.e. Fig. 9). For instance, well before the bulk of the epidemic reaches the Midwest, the disease has already almost reached is maximum in urban areas on the West-Coast. Despite its structural simplicity and the crude assumptions made on the course of deriving the fractional SIS model, these spreading patterns are strikingly similar to recently published large scale agent based simulation studies on the most likely spread of new human influenza H5N1 subtype in the United States.

Although these results are promising, from a theoretical point of view little is known about the general properties of fractional and heterogeneous reaction diffusion equations such as (50) and (51). This is primarily due to the fact that these equations are difficult to solve numerically and the analytical tools for investigation are currently underdeveloped. The richness of possible applications of this approach, not only in spatial epidemiology, however, leads us to believe that in the near future novel and interesting properties of fractional diffusion systems in heterogeneous environments will be discovered and will find their identification in natural systems.

Acknowledgements The authors would like to thank Rafael Brune and Christian Thiemann for their help in improving the manuscript, and Daniel Grady and Olivia Woolley for useful comments.

\section{References}

[1] YC Xia, ON Bjornstad, and BT Grenfell. Measles metapopulation dynamics: A gravity model for epidemiological coupling and dynamics. Am Nat, 164(2):267281, Jan 2004.

[2] http://www.who.int/csr/disease/swineflu.

[3] http://rocs.northwestern.edu/projects/swine_flu. 
[4] L Dall'Asta, A Barrat, M Barthelemy, and A Vespignani. Vulnerability of weighted networks. J Stat Mech-Theory E, page P04006, Jan 2006.

[5] V Colizza, R Pastor-Satorras, and A Vespignani. Reaction-diffusion processes and metapopulation models in heterogeneous networks. Nat Phys, 3(4):276282, Jan 2007.

[6] V Colizza, A Barrat, M Barthelemy, and A Vespignani. The role of the airline transportation network in the prediction and predictability of global epidemics. P Natl Acad Sci Usa, 103(7):2015-2020, Jan 2006.

[7] A Barrat, M Barthelemy, and A Vespignani. The effects of spatial constraints on the evolution of weighted complex networks. J Stat Mech-Theory E, page P05003, Jan 2005.

[8] JV Noble. Geographic and temporal development of plagues. Nature, 250(5469):726-728, Jan 1974

[9] BT Grenfell, ON Bjornstad, and J Kappey. Travelling waves and spatial hierarchies in measles epidemics. Nature, 414(6865):716-723, Jan 2001.

[10] BT Grenfell, ON Bjornstad, and BF Finkenstadt. Dynamics of measles epidemics: Scaling noise, determinism, and predictability with the tsir model. Ecol Monogr, 72(2):185-202, Jan 2002.

[11] NM Ferguson, DAT Cummings, S Cauchemez, C Fraser, S Riley, A Meeyai, S Iamsirithaworn, and DS Burke. Strategies for containing an emerging influenza pandemic in southeast asia. Nature, 437(7056):209-214, Jan 2005.

[12] NM Ferguson, DAT Cummings, C Fraser, JC Cajka, PC Cooley, and DS Burke. Strategies for mitigating an influenza pandemic. Nature, 442(7101):448-452, Jan 2006.

[13] MC Gonzalez, CA Hidalgo, and AL Barabasi. Understanding individual human mobility patterns. Nature, 453(7196):779-782, Jan 2008.

[14] R Guimera and LAN Amaral. Modeling the world-wide airport network. Eur Phys J B, 38(2):381-385, Jan 2004.

[15] L Hufnagel, D Brockmann, and T Geisel. Forecast and control of epidemics in a globalized world. P Natl Acad Sci Usa, 101(42):15124-15129, Jan 2004.

[16] D Brockmann, L Hufnagel, and T Geisel. The scaling laws of human travel. Nature, 439(7075):462-465, Jan 2006.

[17] CW Gardiner. Handbook of Stochastic Methods. Springer Verlag, Berlin, 1985.

[18] R Metzler and J Klafter. The random walks guide to anomalous diffusion: A fractional dynamics approach. Phys. Rep., 339:1-77, 2000. 
[19] MF Shlesinger, GM Zaslavsky, and U Frisch, editors. Lévy Flights and Related Topics in Physics, Lecture Notes in Physics, Berlin, 1995. Springer Verlag.

[20] RM May and RM Anderson. Population biology of infectious-diseases .2. Nature, 280(5722):455-461, Jan 1979.

[21] WO Kermack and AG McKendrick. Contributions to the mathematical theory of epidemics ii - the problem of endemicity. $P R$ Soc Lond A-Conta, 138(834):55-83, Jan 1932.

[22] V Colizza and A Vespignani. Epidemic modeling in metapopulation systems with heterogeneous coupling pattern: Theory and simulations. J Theor Biol, 251(3):450-467, Jan 2008.

[23] VV Belik and D Brockmann. Accelerating random walks by disorder. New $J$ Phys, 9:54, Jan 2007.

[24] D Brockmann and IM Sokolov. Lévy flights in external force fields: From models to equations. Chem. Phys., 284:409-421, 2002.

[25] D Brockmann and T Geisel. Particle dispersion on rapidly folding random hetero-polymers. Phys. Rev. Lett., 91:048303, 2003.

[26] Dirk Brockmann and Theo Geisel. Lévy flights in inhomogeneous media. Phys. Rev. Lett., 90(17):170601, 2003.

[27] E Barkai, R Metzler, and J Klafter. From continuous time random walks to the fractional Fokker-Planck equation. Phys. Rev. E, 61(1):132-138, 2000.

[28] E Barkai. Fractional Fokker-Planck equation, solution, and application. Phys. Rev. E, 63:46118, 2001.

[29] AI Saichev and GM Zaslavsky. Fractional kinetic equations: solutions and applications. Chaos, 7(4):753-764, Jan 1997.

[30] R Metzler and J Klafter. Boundary value problems for fractional diffusion equations. Physica A, 278:107-125, 2000.

[31] D Brockmann and T Geisel. The ecology of gaze shifts. Neurocomputing, 32-33:643-650, 2000. 